\title{
STABILITY OF OPTIMAL-ORDER APPROXIMATION BY BIVARIATE SPLINES OVER ARBITRARY TRIANGULATIONS
}

\author{
C. K. CHUI, D. HONG, AND R. Q. JIA
}

\begin{abstract}
Let $\Delta$ be a triangulation of some polygonal domain in $\mathbb{R}^{2}$ and $S_{k}^{r}(\Delta)$, the space of all bivariate $C^{r}$ piecewise polynomials of total degree $\leq k$ on $\Delta$. In this paper, we construct a local basis of some subspace of the space $S_{k}^{r}(\Delta)$, where $k \geq 3 r+2$, that can be used to provide the highest order of approximation, with the property that the approximation constant of this order is independent of the geometry of $\Delta$ with the exception of the smallest angle in the partition. This result is obtained by means of a careful choice of locally supported basis functions which, however, require a very technical proof to justify their stability in optimal-order approximation. A new formulation of smoothness conditions for piecewise polynomials in terms of their B-net representations is derived for this purpose.
\end{abstract}

\section{INTRODUCTION}

The objective of this paper is to describe the approximation properties of certain bivariate spline spaces over arbitrary triangulations of a polygonal domain in $\mathbb{R}^{2}$ and to construct the approximants that achieve the highest order of approximation. Let $\Delta$ be a 2-dimensional simplicial complex [9, p. 131]. We assume throughout that $\Delta$ is pure; that is, each maximal simplex has dimension 2. Then $\Delta$ is called a triangulation of a polygonal region in $\mathbb{R}^{2}$. As usual, for any nonnegative integers $k$ and $r, S_{k}^{r}(\Delta)$ denotes the space of all $C^{r}$ functions which are piecewise polynomials of total degree at most $k$ separated by $\Delta$. The approximation order of the space $S_{k}^{r}(\Delta)$ is defined to be the largest integer $\rho$ for which

$$
\operatorname{dist}\left(f, S_{k}^{r}(\Delta)\right) \leq C|\Delta|^{\rho}
$$

holds for all sufficiently smooth functions $f$, where the smallest constant $C$, called the approximation constant (of optimal-order), depends only on $f$ and the smallest angle in $\Delta$. Also $|\Delta|:=\sup _{\tau \in \Delta}$ diam $\tau$ denotes the mesh size of $\Delta$, and the distance is measured in the supremum norm \|\| . It is clear

Received by the editors June 22, 1993 and, in revised form, April 4, 1994.

1991 Mathematics Subject Classification. Primary 41A25, 41A63; Secondary 41A05, 41A15, $65 \mathrm{D} 07$.

Key words and phrases. Bivariate splines, triangulations, B-net representations, approximation order, local bases, stability.

Research of the first and second authors supported by NSF Grant No. DMS 92-06928 and ARO Contract DAAH 04-93-G-0047.

Research of the third author supported in part by NSERC Canada under Grant OGP 121336. 
that the approximation order of $S_{k}^{r}(\Delta)$ cannot be higher than $k+1$, regardless of $r$, and is trivially $k+1$ in case $r=0$. On the other hand, it is also well known that for $r \geq 1$ the approximation order from $S_{k}^{r}(\Delta)$ not only depends on $k$ and $r$, but also on the geometric structure of the partition $\Delta$. According to the well-known results in finite element theory (cf. [11]), the full approximation order of $k+1$ is obtained provided that $k \geq 4 r+1$. Extension of this property of optimal approximation to $k \geq 3 r+2$ is more recent. An abstract proof based on the Hahn-Banach theorem was given by de Boor and Höllig [2]. However, as was already pointed out by de Boor [1] (see also Schumaker [10, p. 547]), the proof given in [2] does not fully support the claim that the approximation constant in (1) depends only on the smallest angle in the triangulation. Although constructive proofs were also given in [5] and [6], yet the behavior of the approximation constants still depends on the measurement of "near-singularity" of $\Delta$; i.e., the constant becomes large for near-singular vertices. Observe that when $\Delta$ is refined so that $|\Delta| \rightarrow 0$ in (1), the standard refinement algorithms are mainly concerned with the smallest angle in the partitions, but not with the "near-singularity" of such refinement. Therefore, it is important to give an approximation scheme in order to show that the spline space $S_{k}^{r}(\Delta), k \geq 3 r+2$, admits optimal approximation order of $k+1$ in such a way that the approximation constant $C$ in (1) does not depend on the geometry (such as near-singularity), with the exception of the smallest angle in $\Delta$.

The main purpose of this paper is to construct a stable local basis of the super spline subspace $S_{k}^{r, \mu}(\Delta)$ of $S_{k}^{r}(\Delta)$, where $k \geq 3 r+2$ and $\mu=\left\lfloor\frac{3 r+1}{2}\right\rfloor$ (see [4, p. 73] and [10]), and to show that the full order of approximation can be achieved via a quasi-interpolation scheme using this basis, and that the approximation constant $C$ in (1) of this optimal order depends only on the smallest angle in the triangulation $\Delta$. Unlike the techniques introduced in [2] (see also [1]), which are based on determining the smoothness conditions in terms of the domain points on two triangles that share a common edge to "disentangle the rings" of smoothness conditions, our approach is to inductively determine the smoothness conditions on the rings of the domain points of all vertices; that is, we determine the smoothness conditions in terms of the points on all of the triangles attached to a common vertex.

This paper is organized as follows. In Section 2, in order to facilitate our procedure of constructing a stable super spline basis, we give a new formulation of the smoothness conditions in terms of the B-net representations. In Section 3, we demonstrate how to choose a minimum determining set and provide an explicit scheme of approximation from $S_{k}^{r, \mu}(\Delta)$ that attains the optimal approximation order. Finally, in Section 4 , we will give an explicit scheme for constructing some stable local basis of $S_{k}^{r, \mu}(\Delta)$.

\section{Preliminaries}

Throughout this paper, we will always assume, without loss of generality, that $\Delta$ is connected. For a vertex $v$ of $\Delta$, we denote by $\overline{S t}(v)$ the closed star of vertex $v$ in $\Delta[9$, p. 135]; i.e., the cell formed by all the triangles in $\Delta$ with $v$ as a common vertex. If $\overline{S t}(v) \backslash\{v\}$ is connected for every vertex $v$ of $\Delta$, then $\Delta$ is called strongly connected. If $\Delta$ is strongly connected, then each boundary 
vertex has exactly two boundary edges attached to it. For simplicity, we will always assume that $\Delta$ is strongly connected, though our discussion is also valid otherwise.

Let $\tau=[u, v, w]$ be a triangle with vertices $u, v$ and $w$. For any $x \in \mathbb{R}^{2}$, denote by $\xi(x)=\left(\xi_{u}, \xi_{v}, \xi_{w}\right)$ the barycentric coordinates of $x$ with respect to $\tau$; that is,

$$
x=\xi_{u} u+\xi_{v} v+\xi_{w} w, \quad \xi_{u}+\xi_{v}+\xi_{w}=1 .
$$

For $\alpha=\left(\alpha_{u}, \alpha_{v}, \alpha_{w}\right) \in \mathbb{Z}_{+}^{3}$, the Bernstein-Bézier polynomial $B_{\alpha, \tau}$ is defined by

$$
B_{\alpha, \tau}(x)=\left(\begin{array}{c}
|\alpha| \\
\alpha
\end{array}\right) \xi_{u}^{\alpha_{u}} \xi_{v}^{\alpha_{v}} \xi_{w}^{\alpha_{w}}
$$

where $|\alpha|=\alpha_{u}+\alpha_{v}+\alpha_{w}$ and $\left(\begin{array}{c}|\alpha| \\ \alpha\end{array}\right)=\frac{|\alpha| !}{\alpha_{u} ! \alpha_{v} ! \alpha_{w} !}$. Moreover, we define the points $x_{\alpha, \tau}$ on $\tau$ to be $\left(\alpha_{u} u+\alpha_{v} v+\alpha_{w} w\right) /|\alpha|$. It is well-known that any $p \in \pi_{k}$, the space of all polynomials of total degree $\leq k$, can be written in a unique way as

$$
p=\sum_{|\alpha|=k} b_{\alpha, \tau} B_{\alpha, \tau} .
$$

This gives rise to a mapping $b: x_{\alpha, \tau} \mapsto b_{\alpha, \tau},|\alpha|=k$, and this mapping is called the B-net representation of $p$ with respect to $\tau$.

Let $X$ be the collection $\left\{x_{\alpha, \tau}: \tau \in \Delta,|\alpha|=k\right\}$. To any $f \in S_{k}^{0}(\Delta)$ there corresponds a unique mapping $b_{f}$ from $X$ to $\mathbb{R}$ such that on each $\tau \in \Delta$,

$$
\left.f\right|_{\tau}=\sum_{|\alpha|=k} b_{f}\left(x_{\alpha, \tau}\right) B_{\alpha, \tau} .
$$

This $b_{f}$ is called the B-net representation of $f$ with respect to $\Delta$.

In our investigation, it is essential to represent $C^{r}$-smoothness conditions of spline functions in terms of B-net representations. Suppose that a spline function $f$ is defined over two triangles, say $\tau=[u, v, w]$ and $\tilde{\tau}=[u, v, \tilde{w}]$, with a common edge $[u, v]$. Let $S, S_{u}, S_{v}$ and $S_{w}$ denote the oriented areas of the triangles $\tau,[\tilde{w}, v, w],[u, \tilde{w}, w]$, and $\tilde{\tau}$, respectively. For instance, if $u$ is the origin of $\mathbb{R}^{2}, v=\left(v_{1}, v_{2}\right), w=\left(w_{1}, w_{2}\right)$ and $\tilde{w}=\left(\tilde{w}_{1}, \tilde{w}_{2}\right)$, then

$$
S=\frac{1}{2}\left(v_{1} w_{2}-v_{2} w_{1}\right)
$$

and

$$
S_{v}=\frac{1}{2}\left(\tilde{w}_{1} w_{2}-\tilde{w}_{2} w_{1}\right), S_{w}=\frac{1}{2}\left(v_{1} \tilde{w}_{2}-v_{2} \tilde{w}_{1}\right) .
$$

The following lemma describes $C^{r}$-smoothness conditions on a spline function $f$ in terms of its B-net representation (cf. [8]).

Lemma 1. Suppose that a function $f$ is defined on $\tau \cup \tilde{\tau}$ by

$$
\begin{aligned}
& \left.f\right|_{\tau}=\sum_{|\alpha|=k} b\left(x_{\alpha, \tau}\right) B_{\alpha, \tau} ; \\
& \left.f\right|_{\tilde{\tau}}=\sum_{|\alpha|=k} b\left(x_{\alpha, \tilde{\tau}}\right) B_{\alpha, \tilde{\tau}} .
\end{aligned}
$$


Then $f \in C^{r}(\tau \cup \tilde{\tau})$ if and only if for all nonnegative integers $\ell \leq r$ and $\gamma=\left(\gamma_{u}, \gamma_{v}, 0\right) \in \mathbb{Z}_{+}^{3}$ with $|\gamma|=k-\ell$,

$$
b\left(x_{\gamma+\ell e^{3}, \tilde{\tau}}\right)=\sum_{|\beta|=\ell} b\left(x_{\gamma+\beta, \tau}\right)\left(\begin{array}{l}
\ell \\
\beta
\end{array}\right)\left(\frac{S_{u}}{S}\right)^{\beta_{u}}\left(\frac{S_{v}}{S}\right)^{\beta_{v}}\left(\frac{S_{w}}{S}\right)^{\beta_{w}},
$$

where $\beta=\left(\beta_{u}, \beta_{v}, \beta_{w}\right) \in \mathbb{Z}_{+}^{3}$ and $e^{3}=(0,0,1)$.

We remark that the quantities $\frac{S_{u}}{S}, \frac{S_{v}}{S}$, and $\frac{S_{v}}{S}$ are all bounded by some constant which depends only on the smallest angle in the partition $\Delta$. Hence, as we will see, the approximation constant for optimal-order approximation has to depend on this smallest angle.

For later reference, we need another form of the smoothness conditions which plays an important role to prove the stability of the local basis. For $\alpha=\left(\alpha_{u}, \alpha_{v}, \alpha_{w}\right) \in \mathbb{Z}_{+}^{3}$ with $|\alpha|=k$, let

$$
C_{\alpha, \tau}:=\sum_{\beta_{v}=0}^{\alpha_{v}} \sum_{\beta_{w}=0}^{\alpha_{w}}(-1)^{\alpha_{v}-\beta_{v}+\alpha_{w}-\beta_{w}}\left(\begin{array}{c}
\alpha_{v} \\
\beta_{v}
\end{array}\right)\left(\begin{array}{c}
\alpha_{w} \\
\beta_{w}
\end{array}\right) b\left(x_{\left(k-\beta_{v}-\beta_{w}, \beta_{v}, \beta_{w}\right), \tau}\right) .
$$

Then we have the following result.

Lemma 2. A function $s \in S_{k}^{0}(\tau \cup \tilde{\tau})$ is in $C^{r}$ if and only if the corresponding terms $\left\{C_{\alpha, \tau}\right\}$ and $\left\{C_{\alpha, \tilde{\tau}}\right\}$ satisfy the conditions:

$$
C_{\alpha, \tilde{\tau}}=\sum_{\ell=0}^{\alpha_{w}} C_{\left(\alpha_{u}, \alpha_{v}+\ell, \alpha_{w}-\ell\right), \tau}\left(\begin{array}{c}
\alpha_{w} \\
\ell
\end{array}\right) \frac{S_{v}^{\ell} S_{w}^{\alpha_{w}-\ell}}{S^{\alpha_{w}}},
$$

for $1 \leq \alpha_{w} \leq r$ where $\alpha=\left(\alpha_{u}, \alpha_{v}, \alpha_{w}\right) \in \mathbb{Z}_{+}^{3}$ with $|\alpha|=k$.

Proof. Without loss of generality, we may assume that $u$ is the origin of $\mathbb{R}^{2}$. Let $\tau=[u, v, w], \tilde{\tau}=[u, v, \tilde{w}]$, and consider an $s \in S_{k}^{0}(\tau \cup \tilde{\tau})$ which agrees with some $p \in \pi_{k}$ on $\tau$ and $\tilde{p} \in \pi_{k}$ on $\tilde{\tau}$. For $0 \leq m \leq k$, let $p_{m}$ and $\tilde{p}_{m}$ be the homogeneous components of degree $m$ of $p$ and $\tilde{p}$, respectively. Also, let $s_{m}$ be the corresponding piecewise polynomial function which agrees with $p_{m}$ on $\tau$ and $\tilde{p}_{m}$ on $\tilde{\tau}$. Clearly, $s_{m} \in S_{k}^{0}(\tau \cup \tilde{\tau}), m=0,1, \ldots, k$. Moreover, since we may assume that the mesh line $[u, v]$ is on the $x$-axis, it is not difficult to see that $s$ is in $C^{r}$ if and only if each $s_{m}$ is in $C^{r}, m=0,1, \ldots, k$. Note that

$$
p(x)=\sum_{\nu_{v}+\nu_{w} \leq k} b\left(x_{\nu, \tau}\right) \frac{k !}{\left(k-\nu_{v}-\nu_{w}\right) ! \nu_{v} ! \nu_{w} !}\left(1-\xi_{v}-\xi_{w}\right)^{k-\nu_{v}-\nu_{w}} \xi_{v}^{\nu_{v}} \xi_{w}^{\nu_{w}},
$$

where $\left(1-\xi_{v}-\xi_{w}, \xi_{v}, \xi_{w}\right)$ 1s the barycentric coordinate of $x$ with respect to the triangle $\tau=[u, v, w]$. By the multinomial theorem, we have

$$
\begin{aligned}
& \left(1-\xi_{v}-\xi_{w}\right)^{k-\nu_{v}-\nu_{w}} \\
& \quad=\sum_{\delta_{v}+\delta_{w} \leq k-\nu_{v}-\nu_{w}} \frac{\left(k-\nu_{v}-\nu_{w}\right) !}{\left(k-\nu_{v}-\nu_{w}-\delta_{v}-\delta_{w}\right) ! \delta_{v} ! \delta_{w} !}(-1)^{\delta_{v}+\delta_{w}} \xi_{v}^{\delta_{v}} \xi_{w}^{\delta_{w}} .
\end{aligned}
$$

Therefore, by setting $\delta_{v}+\nu_{v}$ and $\delta_{w}+\nu_{w}$ to be $\alpha_{v}$ and $\alpha_{w}$, respectively, we 
have

$$
\begin{aligned}
p(x)=\sum_{\alpha_{v}+\alpha_{w} \leq k} \sum_{\substack{\nu_{v} \leq \alpha_{v} \\
\nu_{w} \leq \alpha_{w}}} b\left(x_{\nu, \tau}\right) \frac{k !}{\left(k-\alpha_{v}-\alpha_{w}\right) ! \alpha_{v} ! \alpha_{w} !} \\
\cdot\left(\begin{array}{c}
\alpha_{v} \\
\nu_{v}
\end{array}\right)\left(\begin{array}{c}
\alpha_{w} \\
\nu_{w}
\end{array}\right)(-1)^{\alpha_{v}-\nu_{v}+\alpha_{w}-\nu_{w}} \xi_{v}^{\alpha_{v}} \xi_{w}^{\alpha_{w}} .
\end{aligned}
$$

Recall that $u=(0,0)$. So, writing $v=\left(v_{1}, v_{2}\right), w=\left(w_{1}, w_{2}\right)$, and $x=$ $\left(x_{1}, x_{2}\right)$, we see that

$$
\xi_{v}=\frac{\operatorname{area}[u, x, w]}{\operatorname{area}[u, v, w]}=\frac{x_{1} w_{2}-x_{2} w_{1}}{v_{1} w_{2}-v_{2} w_{1}}
$$

and

$$
\xi_{w}=\frac{\operatorname{area}[u, v, x]}{\operatorname{area}[u, v, w]}=\frac{v_{1} x_{2}-v_{2} x_{1}}{v_{1} w_{2}-v_{2} w_{1}}
$$

are homogeneous linear functions of $x$; hence, we have

$$
\begin{aligned}
p_{m}(x)=\sum_{\alpha_{v}+\alpha_{w}=m} \sum_{\substack{\nu_{v} \leq \alpha_{v} \\
\nu_{w} \leq \alpha_{w}}} b\left(x_{\nu, \tau}\right) \frac{k !}{(k-m) ! \alpha_{v} ! \alpha_{w} !} \\
\cdot\left(\begin{array}{c}
\alpha_{v} \\
\nu_{v}
\end{array}\right)\left(\begin{array}{c}
\alpha_{w} \\
\nu_{w}
\end{array}\right)(-1)^{\alpha_{v}-\nu_{v}+\alpha_{w}-\nu_{w}} \xi_{v}^{\alpha_{v}} \xi_{w}^{\alpha_{w}} .
\end{aligned}
$$

Taking (5) into account, we deduce that

$$
p_{m}(x)=\sum_{\alpha_{v}+\alpha_{w}=m} \frac{k !}{(k-m) ! \alpha_{v} ! \alpha_{w} !} C_{\alpha, \tau} \xi_{v}^{\alpha_{v}} \xi_{w}^{\alpha_{w}} .
$$

Similarly, we have

$$
\tilde{p}_{m}(x)=\sum_{\alpha_{v}+\alpha_{\tilde{w}}=m} \frac{k !}{(k-m) ! \alpha_{v} ! \alpha_{\tilde{w}} !} C_{\alpha, \tilde{\tau}} \tilde{\xi}_{v}^{\alpha_{v}} \tilde{\xi}_{\tilde{w}}^{\alpha_{\tilde{w}}},
$$

where $\left(1-\tilde{\xi}_{v}-\tilde{\xi}_{\tilde{w}}, \tilde{\xi}_{v}, \tilde{\xi}_{\tilde{w}}\right)$ is the barycentric coordinate of $x$ with respect to $[u, v, \tilde{w}]$.

Let us now express $\xi_{v}$ and $\xi_{w}$ in terms of $\tilde{\xi}_{v}$ and $\tilde{\xi}_{\tilde{w}}$. Suppose $\tilde{w}=$ $\left(\tilde{w}_{1}, \tilde{w}_{2}\right)$. Then we have

$$
\tilde{\xi}_{v}=\frac{x_{1} \tilde{w}_{2}-x_{2} \tilde{w}_{1}}{v_{1} \tilde{w}_{2}-v_{2} \tilde{w}_{1}}, \quad \tilde{\xi}_{\tilde{w}}=\frac{v_{1} x_{2}-v_{2} x_{1}}{v_{1} \tilde{w}_{2}-v_{2} \tilde{w}_{1}}
$$

These two equalities together with (2) and (3) yield

$$
\xi_{w}=\frac{S_{w}}{S} \tilde{\xi}_{\tilde{w}}
$$

and

$$
\xi_{v}=\tilde{\xi}_{v}+\frac{S_{v}}{S} \tilde{\xi}_{\tilde{w}}
$$


Finally, replacing $\xi_{v}$ by $\tilde{\xi}_{v}+\frac{S_{v}}{S} \tilde{\xi}_{\tilde{w}}$ and $\xi_{w}$ by $\frac{S_{w}}{S} \tilde{\xi}_{\tilde{w}}$ in (7), we obtain

$$
\begin{gathered}
p_{m}(x)=\sum_{\alpha_{v}+\alpha_{w}=m} \frac{k !}{(k-m) ! \alpha_{v} ! \alpha_{w} !} C_{\alpha, \tau}\left(\tilde{\xi}_{v}+\frac{S_{v}}{S} \tilde{\xi}_{\tilde{w}}\right)^{\alpha_{v}}\left(\frac{S_{w}}{S} \tilde{\xi}_{\tilde{w}}\right)^{\alpha_{w}} \\
=\sum_{\alpha_{v}+\alpha_{w}=m} \sum_{\ell=0}^{\alpha_{v}} \frac{k !}{(k-m) ! \alpha_{v} ! \alpha_{w} !} \frac{\alpha_{v} !}{\ell !\left(\alpha_{v}-\ell\right) !} C_{\alpha, \tau} \\
\cdot\left(\frac{S_{v}}{S}\right)^{\alpha_{v}-\ell}\left(\frac{S_{w}}{S}\right)^{\alpha_{w}} \tilde{\xi}_{v}^{\ell} \tilde{\xi}_{\tilde{w}}^{\alpha_{v}-\ell+\alpha_{w}} .
\end{gathered}
$$

Let $\beta_{\tilde{w}}=\alpha_{v}+\alpha_{w}-\ell, \beta_{v}=\ell$ and $q=\alpha_{v}-\ell$. Then we have $\alpha_{w}=\beta_{\tilde{w}}-q$, and

$$
p_{m}(x)=\sum_{\beta_{v}+\beta_{\dot{w}=m}} \frac{k !}{(k-m) ! \beta_{v} ! \beta_{\tilde{w}} !} \sum_{q=0}^{\beta_{\tilde{w}}}\left(\begin{array}{c}
\beta_{\tilde{w}} \\
q
\end{array}\right) C_{\left(k-m, \beta_{v}+q, \beta_{\tilde{w}}-q\right), \tau} \frac{S_{v}^{q} S_{w}^{\beta_{\tilde{w}}-q}}{S^{\beta_{\tilde{w}}}} \tilde{\xi}_{v}^{\beta_{v}} \tilde{\xi}_{\tilde{w}}^{\beta_{\tilde{w}}}
$$

Comparing this with the expression for $\tilde{p}_{m}(x)$ in (8), we conclude that $s_{m} \in C^{r}$ if and only if

$$
C_{\beta, \tilde{\tau}}=\sum_{q=0}^{\beta_{\tilde{w}}}\left(\begin{array}{c}
\beta_{\tilde{w}} \\
q
\end{array}\right) C_{\left(\beta_{u}, \beta_{v}+q, \beta_{\tilde{w}}-q\right), \tau} \frac{S_{v}^{q} S_{w}^{\beta_{\tilde{w}}-q}}{S^{\beta_{\tilde{w}}}}
$$

for $\beta=\left(\beta_{u}, \beta_{v}, \beta_{\tilde{w}}\right) \in \mathbb{Z}_{+}^{3}$ with $1 \leq \beta_{\tilde{w}} \leq r$ and $\beta_{v}+\beta_{\tilde{w}}=m$. This completes the proof of the lemma.

\section{MAIN RESUlts}

To investigate the approximation properties of bivariate splire spaces, it is convenient to introduce the notion of super splines. Given a triangulation $\Delta$ and nonnegative integers $k, r$ and $\mu$ with $k \geq \mu \geq r$, a super spline is a piecewise polynomial of degree at most $k$ on $\Delta$ which is $C^{r}$ across each edge and $C^{\mu}$ around each vertex. Let $S_{k}^{r, \mu}(\Delta)$ be the space of all such splines. Then $S_{k}^{r, \mu}(\Delta)$ is a subspace of $S_{k}^{r}(\Delta)$. In this section, we describe an explicit quasi-interpolation scheme and prove that the super spline space $S_{k}^{r, \mu}(\Delta), \mu=$ $\left\lfloor\frac{3 r+1}{2}\right\rfloor, k \geq 3 r+2$, admits the optimal approximation order of $k+1$ with the approximation constant dependent only on the smallest angle in the partition $\Delta$.

Let us introduce a natural pairing

$$
\langle\lambda, b\rangle:=\sum_{x \in X} \lambda(x) b(x), \quad \lambda, b \in \mathbb{R}^{X},
$$

on $\mathbb{R}^{X}$. Now choose and fix an orientation for each interior edge of $\Delta$. Let $e=$ $[u, v]$ be an oriented interior edge with two triangles $[u, v, w]$ and $[u, v, \tilde{w}]$ attached to it. If the orientation of $e$ is from $u$ to $v$, then we assume that the points $u, v$ and $w$ are ordered in the counterclockwise direction. In this case, we say that the orientation of the triangle $\tau$ agrees with the orientation of the edge $e$. Let $\alpha=\left(\alpha_{u}, \alpha_{v}, \alpha_{\tilde{w}}\right) \in \mathbb{Z}_{+}^{3}$ with $|\alpha|=k$ and $\alpha_{\tilde{w}} \geq 1$. Bearing 
Lemma 1 in mind, we define a function $\lambda_{e, \alpha}$ on $X$ as follows:

$$
\lambda_{e, \alpha}(x):= \begin{cases}1, & \text { if } x=x_{\alpha, \tilde{\tau}} ; \\
-\left(\begin{array}{c}
\alpha_{\tilde{w}} \\
\beta
\end{array}\right) \frac{S_{u}^{\beta_{u}} S_{v}^{\beta_{v}} S_{w}^{\beta_{w}}}{S^{\alpha_{\tilde{w}}}}, & \text { if } x=x_{\left(\alpha_{u}, \alpha_{v}, 0\right)+\beta, \tau} \\
& \text { for } \beta \in \mathbb{Z}_{+}^{3} \text { with }|\beta|=\alpha_{\tilde{w}} \\
0, & \text { elsewhere. }\end{cases}
$$

The points $x_{\alpha, \tau}$ and $x_{\alpha, \tilde{\tau}}$ will be called the tips of $\lambda_{e, \alpha}$.

In the sequel, we always assume that $k \geq 3 r+2$ and consider $\mu:=\left\lfloor\frac{3 r+1}{2}\right\rfloor$. For a vertex $u$ and an oriented interior edge $e$ attached to $u$, we consider the collections $\Lambda_{u, e}^{n}$ defined by

$$
\Lambda_{u, e}^{n}=\left\{\lambda_{e, \alpha}: \alpha_{u}=k-n\right\}, \quad n=1,2, \ldots, \mu,
$$

and

$$
\Lambda_{u, e}^{n}:=\left\{\lambda_{e, \alpha}: \alpha_{u}=k-n ; \alpha_{v}, \alpha_{\tilde{w}} \leq r\right\}, \quad n=\mu+1, \ldots, 2 r .
$$

Furthermore, let

$$
\begin{aligned}
\Lambda_{u}^{n} & :=\bigcup_{e \ni u} \Lambda_{u, e}^{n}, \quad n=1,2, \ldots, 2 r, \\
\Lambda_{u} & :=\bigcup_{n=1}^{2 r} \Lambda_{u}^{n},
\end{aligned}
$$

and for an oriented interior edge $e$, let

$$
\Lambda_{e}:=\left\{\lambda_{e, \alpha}: 1 \leq \alpha_{\tilde{w}} \leq r<\alpha_{u}, \alpha_{v}<k-\mu\right\} .
$$

Finally, let

$$
\Lambda:=\left(\bigcup_{u \in V} \Lambda_{u}\right) \cup\left(\bigcup_{e \in E} \Lambda_{e}\right),
$$

where $V$ and $E$ denote the collections of vertices and oriented interior edges of $\Delta$, respectively. By Lemma 1 , we see that $f \in S_{k}^{r, \mu}(\Delta)$ if and only if its B-net representation $b_{f}$ satisfies

$$
\left\langle\lambda, b_{f}\right\rangle=0, \quad \lambda \in \Lambda \text {. }
$$

A subset $Y$ of $X$ is called a determining set for the super spline space $S_{k}^{r, \mu}(\Delta)$, if the linear mapping $\left.f \mapsto b_{f}\right|_{Y}$ defined on $S_{k}^{r, \mu}(\Delta)$ is one-to-one. Our goal is to find a minimum determining set for this super spline space.

An interior vertex $u$ is said to be singular, if there are exactly four edges attached to it and these edges lie on two straight lines. Otherwise, $u$ is called nonsingular. In particular, a boundary vertex is regarded as nonsingular.

For a vertex $u$ and a triangle $\tau=[u, v, w]$ attached to $u$, let

$$
\begin{gathered}
X_{u, \tau}^{n}:=\left\{x_{\alpha, \tau}: \alpha_{u}=k-n\right\}, \quad n=0,1, \ldots, \mu ; \\
X_{u}^{n}:=\bigcup_{\tau \ni u} X_{u, \tau}^{n}, \quad n=0,1, \ldots, \mu .
\end{gathered}
$$

We associate with each vertex $u$ a triangle $\tau$ attached to $u$ and define

$$
Y_{u}^{n}:=X_{u, \tau}^{n}, \quad n=0,1, \ldots, \mu .
$$




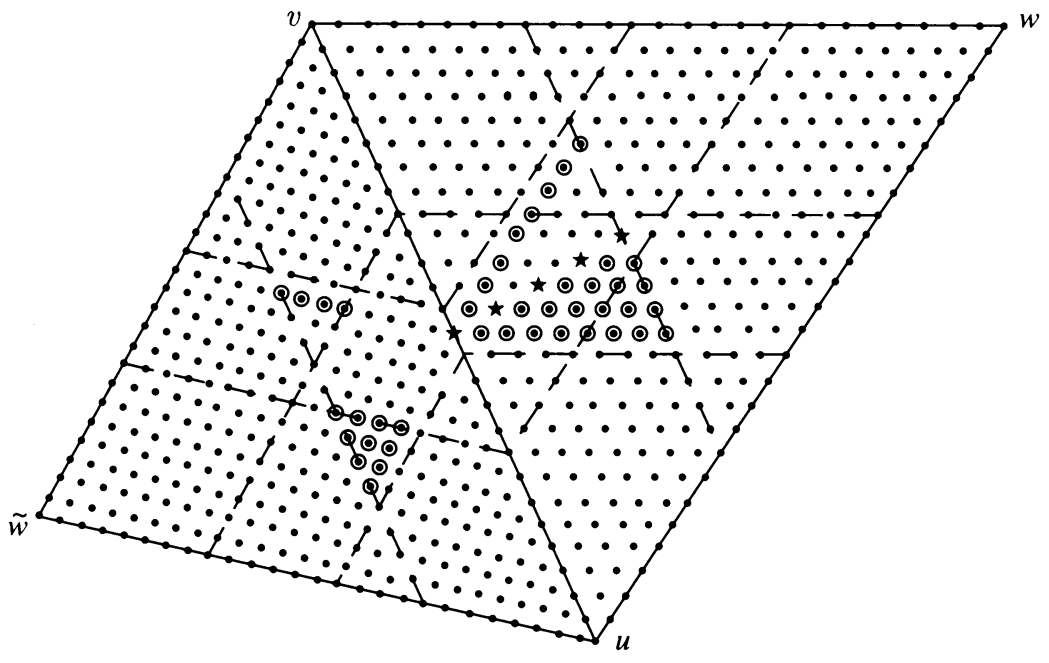

FIGURE 1. The points “ $\odot$ ” in $X_{u, e}^{n}, n=\mu+1, \ldots, 2 r$, and $X_{v, e}^{n}, n=\mu+1$, and " $\star$ " in $Y_{e}$ for nonsingular vertex $u(k=26, r=8, \mu=12)$.

Let $e$ be any oriented interior edge with a given $u$ and some $v$ as two of its vertices. Also let $\tau$ and $\tilde{\tau}$ be the two triangles attached to $e$, such that the orientation of $\tau$ agrees with that of $e$; moreover, denote by $w$ and $\tilde{w}$ the third vertices of $\tau$ and $\tilde{\tau}$, respectively. For $n=\mu+1, \cdots, 2 r$, if $u$ is a nonsingular vertex, we define $X_{u, e}^{n}$ to be the union of the two sets

$$
\left\{x_{\alpha, \tilde{\tau}}: \alpha_{u}=k-n ; n-r \leq \alpha_{\tilde{w}} \leq r\right\}
$$

and

$$
\left\{x_{\alpha, \tau}: \alpha_{u}=k-n ; 2 n-3 r-1 \leq \alpha_{w} \leq r\right\}
$$

(see Figure 1). If $u$ is a singular vertex and $\tau=[u, v, w]$ is a triangle attached to $u$, we define

$$
X_{u, \tau}^{n}:=\left\{x_{\alpha, \tau}: \alpha_{u}=k-n ; n-r \leq \alpha_{w} \leq r\right\}, \quad n=\mu+1, \ldots, 2 r,
$$

(see Figure 2). If $e$ is an oriented edge attached to a singular vertex $u$, we set

$$
X_{u, e}^{n}:=X_{u, \tau}^{n} \cup X_{u, \tau}^{n}, \quad n=\mu+1, \ldots, 2 r,
$$

where $\tau$ and $\tilde{\tau}$ are the two triangles with common edge $e$; also, set

$$
Y_{u}^{n}:=X_{u, \tau}^{n}, \quad n=\mu+1, \ldots, 2 r,
$$

where $\tau$ is an arbitrarily chosen triangle attached to $u$. For any vertex $u$, singular or otherwise, we define

$$
X_{u}^{n}:=\bigcup_{e \ni u} X_{u, e}^{n}, \quad n=\mu+1, \ldots, 2 r .
$$

Furthermore, we associate with each oriented interior edge $e$ three sets

$$
\begin{aligned}
& X_{e}^{+}:=\left\{x_{\alpha, \tau}: 0 \leq \alpha_{w} \leq r<\alpha_{u}, \alpha_{v}<k-\mu\right\}, \\
& X_{e}^{-}:=\left\{x_{c, \dot{\tau}}: 1 \leq \alpha_{\tilde{u}} \leq r<\alpha_{u}, \alpha_{v}<k-\mu\right\},
\end{aligned}
$$




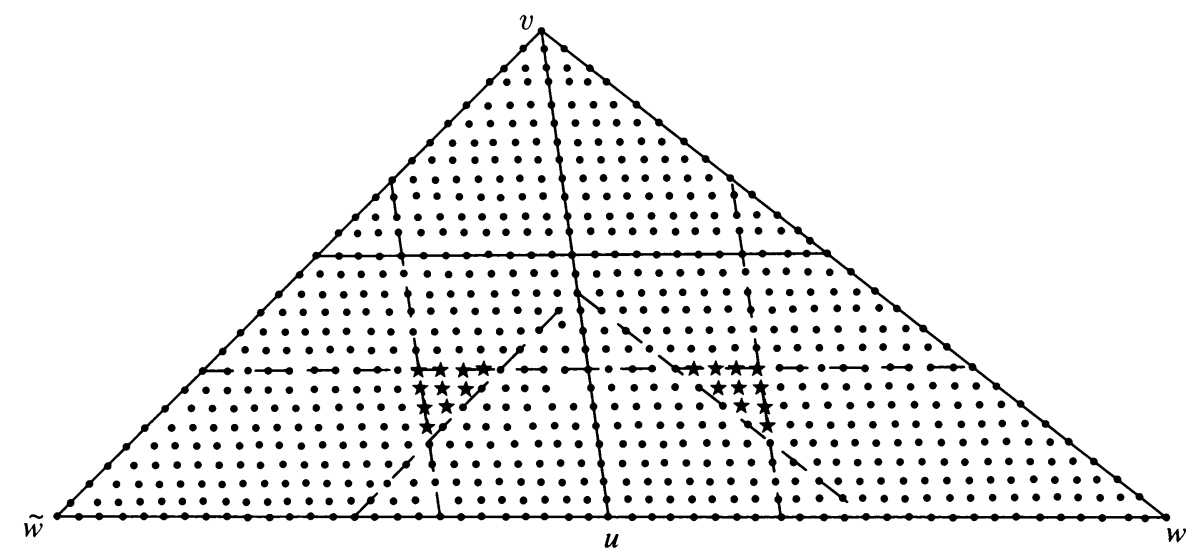

FIGURE 2. The points in $X_{u, e}^{n}, n=\mu+1, \ldots, 2 r$, for a singular vertex $u(k=26, r=8, \mu=12)$.

and

$$
Y_{e}:=X_{e}^{+} \backslash \bigcup_{n=\mu+1}^{2 r}\left(X_{u, e}^{n} \cup X_{v, e}^{n}\right) .
$$

Finally, for each triangle $\tau$, we define

$$
X_{\tau}:=\left\{x_{\alpha, \tau}: \alpha_{u}, \alpha_{v}, \alpha_{w}>r\right\} .
$$

From the preceding construction we see that $X$ is the disjoint union

$$
\left(\bigcup_{n=0}^{2 r} \bigcup_{u \in V} X_{u}^{n}\right) \cup\left(\bigcup_{e \in E}\left(Y_{e} \cup X_{e}^{-}\right)\right) \cup\left(\bigcup_{\tau \in \Gamma} X_{\tau}\right) \text {, }
$$

where $\Gamma$ denotes the collection of all triangles in $\Delta$.

Suppose now that $u$ is a nonsingular vertex. Then for each integer $n$ between $\mu+1$ and $2 r$, we choose a subset $Z_{u}^{n}$ of $X_{u}^{n}$ such that the cardinality $\# Z_{u}^{n}$ of $Z_{u}^{n}$ is equal to $\# \Lambda_{u}^{n}$, and

$$
\left|\operatorname{det}(\lambda(x))_{\lambda \in \Lambda_{u}^{n}, x \in Z_{u}^{n}}\right| \geq\left|\operatorname{det}(\lambda(x))_{\lambda \in \Lambda_{u}^{n}, x \in Z}\right|
$$

for any subset $Z$ of $X_{u}^{n}$ with $\# Z=\# \Lambda_{u}^{n}$. It is known that the matrix $(\lambda(x))_{\lambda \in \Lambda_{u}^{n}, x \in X_{u}^{n}}$ has full (row) rank (see [2, Proposition 6] and [7]); hence

$$
\operatorname{det}(\lambda(x))_{\lambda \in \Lambda_{u}^{n}, x \in Z_{u}^{n}} \neq 0,
$$

and we will write

$$
Y_{u}^{n}:=X_{u}^{n} \backslash Z_{u}^{n}, \quad n=\mu+1, \ldots, 2 r .
$$

For each triangle $\tau \in \Delta$, we define

Finally, we set

$$
Y_{\tau}:=X_{\tau} .
$$

$$
Y_{v}:=\bigcup_{n=0}^{2 r} Y_{v}^{n}
$$




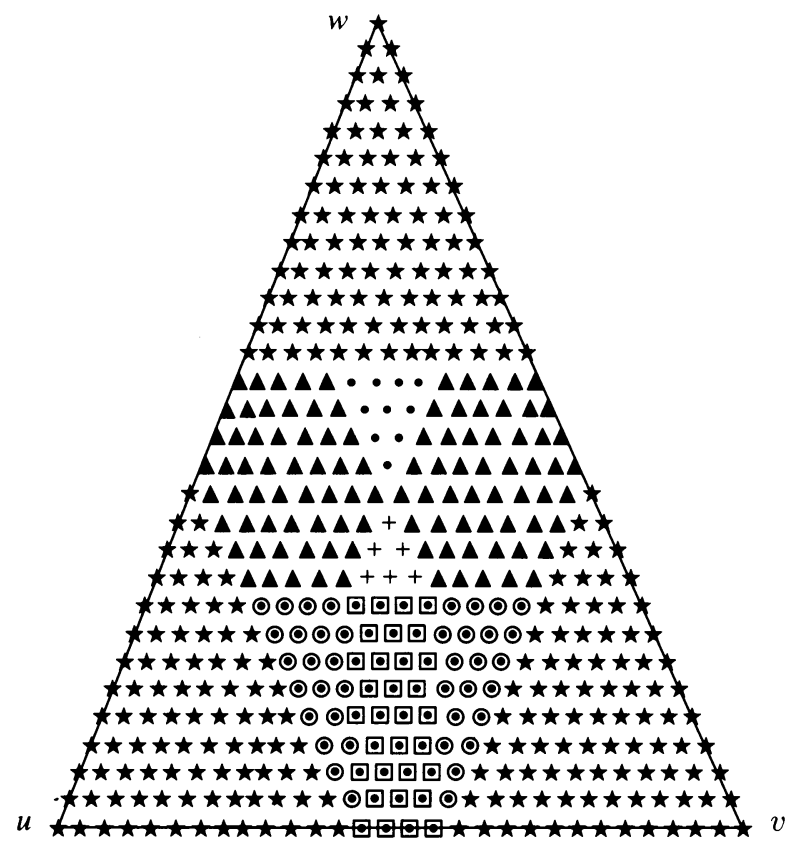

Figure 3. The classification of point set $X$ on a triangle.

and

$$
Y:=\left(\bigcup_{v \in V} Y_{v}\right) \cup\left(\bigcup_{e \in E} Y_{e}\right) \cup\left(\bigcup_{\tau \in \Gamma} Y_{\tau}\right) .
$$

Then from the following theorem, we see that $Y$ is a minimum determining set for $S_{k}^{r, \mu}(\Delta)$.

Theorem 1. For each $b: Y \mapsto \mathbb{R}$, there exists a unique $f \in S_{k}^{r, \mu}(\Delta)$ such that the B-net representation $b_{f}$ of $f$ satisfies

$$
\left.b_{f}\right|_{Y}=b \text {. }
$$

Proof. Let $\Lambda^{\perp}:=\left\{b \in \mathbb{R}^{X}:\langle\lambda, b\rangle=0, \lambda \in \Lambda\right\}$, where $\Lambda$ is given by (14). Then $f \in S_{k}^{r, \mu}(\Delta)$ if and only if $b_{f} \in \Lambda^{\perp}$; hence, it suffices to show that for a given mapping $b: Y \mapsto \mathbb{R}$, there exists a unique $\widehat{b} \in \Lambda^{\perp}$ such that $\left.\widehat{b}\right|_{Y}=b$.

We shall first extend $b$ to $\bigcup_{u \in V} X_{u}^{n}$ for $n=0,1, \ldots, \mu$. For each $u \in V$, (16) tells us that $Y_{u}^{n}=X_{u, \tau}^{n}$ for $n=0,1, \ldots, \mu$, where $\tau$ is a triangle attached to $u$. On the other hand, there exists a polynomial $p_{u} \in \pi_{k}$ such that its B-net representation $b_{p_{u}}$ on $\Delta$ satisfies $b_{p_{u}}(x)=b(x)$ for all $x \in \bigcup_{n=0}^{\mu} Y_{u}^{n}$. We extend $b$ to $\bigcup_{n=0}^{\mu} X_{u}^{n}$ by setting $\widehat{b}(x):=b_{p_{u}}(x)$ for every $x \in \bigcup_{n=0}^{\mu} X_{u}^{n}$. Evidently, $\langle\lambda, \widehat{b}\rangle=0$ for all $\lambda \in \bigcup_{n=1}^{\mu} \Lambda_{u}^{n}$.

Next, we extend $b$ to $\bigcup_{u \in V} X_{u}^{n}$ for $n=\mu+1, \ldots, 2 r$. This is done inductively on $n$ as follows. Let $n$ be given with $\mu+1 \leq n \leq 2 r$. Suppose that $\widehat{b}(x)$ has been determined for $x \in \bigcup_{j=0}^{n-1} \bigcup_{u \in V} X_{u}^{j}$ in such a way that $\langle\lambda, \widehat{b}\rangle=0$ for all $\lambda \in \bigcup_{j=1}^{n-1} \bigcup_{u \in V} \Lambda_{u}^{j}$. We wish to determine the values of $\widehat{b}$ on $\bigcup_{u \in V} X_{u}^{n}$ 
such that for every $u \in V$,

$$
\sum_{x \in X} \lambda(x) \widehat{b}(x)=0, \quad \lambda \in \Lambda_{u}^{n}
$$

We claim that the value $\widehat{b}(x)$ has been determined whenever $x \in X \backslash X_{u}^{n}$ and $\lambda(x) \neq 0$ for some $\lambda \in \Lambda_{u}^{n}$. To establish this claim, we consider $\lambda=\lambda_{e, \alpha}$, where $e$ is an edge attached to $u$ and $\alpha=\left(\alpha_{u}, \alpha_{v}, \alpha_{w}\right)$ with $\alpha_{u}=k-n$. Without loss of generality, we assume that $e=[u, v]$ is an oriented interior edge and both of $u$ and $v$ are nonsingular, for otherwise the proof is analogous. Let $\tau=[u, v, w]$ and $\tilde{\tau}=[u, v, \tilde{w}]$ be the two triangles with common edge $e$. If $\lambda(x) \neq 0$ and $x \notin X_{u}^{n}$, then $x=x_{\beta, \tau}$ for some $\beta \in \mathbb{Z}_{+}^{3}$ with $|\beta|=k$, $\beta_{u} \geq \alpha_{u}, \quad \beta_{v} \geq \alpha_{v}$. Thus, we have $\beta_{u}=k-m$ for some $m \leq n$. Since $\widehat{b}(x)$ has been determined for $x \in \bigcup_{j=0}^{n-1} \bigcup_{u \in V} X_{u}^{j}$, we may assume that $x \notin$ $\bigcup_{j=0}^{n-1} \bigcup_{u \in V} X_{u}^{j}$. It follows that $\mu+1 \leq m \leq 2 r$. By the definition of $X_{u}^{m}$, we have $\beta_{w}<2 m-3 r-1$. Consequently, $x_{\beta, \tau} \notin X_{v}^{p}$ for any $p \geq m$. This shows that

$$
x_{\beta, \tau} \notin \bigcup_{n=\mu+1}^{2 r}\left(X_{u, e}^{n} \cup X_{v, e}^{n}\right) \text {. }
$$

By the definition of $Y_{e}$, we have $x=x_{\beta, \tau} \in Y_{e}$, and therefore $\widehat{b}(x)=b(x)$ is already determined. This verifies our claim.

Suppose now that $u$ is a nonsingular vertex. Remember that $X_{u}^{n}$ is the disjoint union of $Y_{u}^{n}$ and $Z_{n}^{n}$ and $\hat{b}(x)=b(x)$ for $x \in Y_{u}^{n}$. Moreover,

$$
\operatorname{det}(\lambda(x))_{\lambda \in \Lambda_{u}^{n}, x \in Z_{u}^{n}} \neq 0 .
$$

Thus, the values of $\widehat{b}$ on $Z_{u}^{n}$ can be determined by solving the system

$$
\sum_{x \in Z_{u}^{n}} \lambda(x) \hat{b}(x)=-\sum_{x \in X \backslash Z_{u}^{n}} \lambda(x) \hat{b}(x), \quad \lambda \in \Lambda_{u}^{n}
$$

of linear equations.

It remains to deal with the case where $u$ is a singular vertex. Suppose that $\tau_{1}, \tau_{2}, \tau_{3}$ and $\tau_{4}$ are the four triangles attached to $u$ and arranged in a consecutive way. We may assume that $Y_{u}^{n}=X_{u, \tau_{1}}^{n}, n=\mu+1, \ldots, 2 r$. Let $e_{j}$, $j=1,2,3,4$, be the common edge of $\tau_{j}$ and $\tau_{j+1}$ with $\tau_{5}:=\tau_{1}$. We have $\widehat{b}(x)=b(x)$ for $x \in Y_{u}^{n}=X_{u, \tau_{1}}^{n}$. Note that the matrix $(\lambda(x))_{\lambda \in \Lambda_{u, e_{j}}^{n}, x \in X_{u, \tau_{j+1}}^{n}}$ is a nonsingular diagonal matrix if the $\lambda$ 's and the $x$ 's are arranged in an appropriate way. Thus, we can determine the values of $\widehat{b}$ on $X_{u, \tau_{j+1}}^{n}, j=$ $1,2,3$, by solving the system

$$
\sum_{x \in X_{u, \tau_{j+1}}^{n}} \lambda(x) \hat{b}(x)=-\sum_{x \in X \backslash X_{u, \tau_{j+1}}^{n}} \lambda(x) \hat{b}(x), \quad \lambda \in \Lambda_{u, e_{j}}^{n}
$$

of linear equations.

It is known (see, e.g., [7]) that the function $\hat{b}$ so obtained also satisfies

$$
\sum_{x \in X} \lambda(x) \hat{b}(x)=0 \quad \text { for all } \lambda \in \Lambda_{u, e_{4}}^{n}
$$


Finally, we extend $b$ to all the remaining points $X$; i.e., to the points in $\bigcup_{e \in E} X_{e}^{-}$. This can be easily done by applying the smoothness conditions (4) across each interior edge.

To summarize, we have constructed a function $\widehat{b}$ on $X$ such that $\widehat{b} \in \Lambda^{\perp}$ and $\left.\widehat{b}\right|_{Y}=b$. From this construction, we see that such a $\widehat{b}$ is unique. Indeed, if $b=0$ on $Y$ and $\widehat{b} \in \Lambda^{\perp}$ satisfies $\left.\widehat{b}\right|_{Y}=b=0$, then $\widehat{b}$ must vanish on all of $X$. This completes the proof of the theorem.

Theorem 1 suggests the following Approximation Scheme.

Step 1. Given $f \in C(\Delta)$ and a triangle $\tau \in \Delta$, find $p_{\tau} \in \pi_{k}$ such that

$$
p_{\tau}\left(x_{\alpha, \tau}\right)=f\left(x_{\alpha, \tau}\right) \text { for all }|\alpha|=k .
$$

Step 2. Let $s \in S_{k}^{0}(\Delta)$ be the spline function given by

$$
\left.s\right|_{\tau}=p_{\tau}
$$

for each triangle $\tau \in \Delta$. Then find the B-net representation $b$ of $s$.

Step 3. Find $\widehat{b}$ in accordance with Theorem 1 , such that $\left.\widehat{b}\right|_{Y}=\left.b\right|_{Y}$ and $\widehat{b} \in$ $\Lambda^{\perp}$. Let $g$ be the spline in $S_{k}^{r, \mu}(\Delta)$ whose B-net representation $b_{g}$ agrees with $\widehat{b}$ on $X$.

We denote by $T$ the linear operator $f \mapsto g, f \in C(\Delta)$.

In the sequel, we will denote by $a$ the smallest angle in $\Delta$, and by Const $_{a, k}$ we mean a constant depending only on $a$ and $k$, which may vary from situation to situation. We use the notation $D_{j}, j=1,2$, to denote the partial derivative operators with respect to the $j$ th coordinates. Also, the closed star of $v$, denoted by $\overline{S t}(v)=: \overline{S t}^{1}(v)$, is the union of all the triangles attached to $v$, and the $m$-star of $v$, denoted by $\overline{S t}^{m}(v)$, is the union of all triangles that intersect with $\overline{S t}^{m-1}(v), m>1$.

Lemma 3. The linear operator $T$ satisfies the following conditions:

(i) $T p=p$ for every polynomial $p \in \pi_{k}$.

(ii) If $\tau$ is a triangle attached to a vertex $u$, then

$$
\left\|\left.(T f)\right|_{\tau}\right\|_{\infty} \leq \text { Const }_{a, k}\left\|\left.f\right|_{N(u)}\right\|_{\infty},
$$

where $N(u)$ denotes the star $\overline{S t}^{\lfloor r / 2\rfloor+2}(u)$.

Proof. The first part of this lemma is a straightforward consequence of the construction of $T$. The second part will be proved in the next section.

We are now in a position to establish the main result of this paper.

Theorem 2. If $k \geq 3 r+2$, then there exists a linear operator $T$ from $C^{k+1}(\Delta)$ to $S_{k}^{r, \mu}(\Delta)$ such that

$$
\|f-T f\|_{\infty} \leq \text { Const }_{a, k}|\Delta|^{k+1}|f|_{k+1, \infty},
$$

where $|f|_{k+1, \infty}:=\sum_{\gamma_{1}+\gamma_{2}=k+1}\left\|D_{1}^{\gamma_{1}} D_{2}^{\gamma_{2}} f\right\|_{\infty}$.

Proof. Let $T$ be the operator described by the above approximation scheme. Let $f \in C^{k+1}(\Delta)$ be given. In order to estimate the error $f-T f$, we consider 
$f(x)-(T f)(x)$, where $x$ is a point in a triangle $\tau$ of $\Delta$. Then there exists a polynomial $p \in \pi_{k}$ such that $p(x)=f(x)$ and

$$
|p(y)-f(y)| \leq \text { Const }_{k}|f|_{k+1, \infty}|\Delta|^{k+1} \text { for all } y \in N(u) .
$$

By Lemma 3, we deduce from (24) that

$$
\begin{aligned}
|f(x)-T f(x)| & =|T(f-p)(x)| \leq\left\|\left.T(f-p)\right|_{\tau}\right\|_{\infty} \\
& \leq \text { Const }_{a, k}\left\|\left.(f-p)\right|_{N(u)}\right\|_{\infty} \leq \text { Const }_{a, k}|f|_{k+1, \infty}|\Delta|^{k+1} .
\end{aligned}
$$

This estimate is valid for every $x \in \Delta$. Hence, the proof of the theorem is complete.

\section{STABle Bases With LOCAL SUPPORT}

In this section, by using the determining set as described in Section 3, we shall construct a basis for $S_{k}^{r, \mu}(\Delta)$ which is both stable and local.

To begin with, we establish the following result about the norm estimation of the B-net ordinates of any function in $S_{k}^{r, \mu}(\Delta)$.

Theorem 3. Every $b \in \mathbb{R}^{X} \cap \Lambda^{\perp}$ satisfies

$$
\|b\|_{\infty} \leq \text { Const }_{a, k}\left\|\left.b\right|_{Y}\right\|_{\infty},
$$

where $Y$ is the determining set for $S_{k}^{r, \mu}(\Delta)$ as defined by (21).

Proof. Let $M:=\left\|\left.b\right|_{Y}\right\|_{\infty}$. First, we show that, for $n=0,1, \ldots, \mu$,

$$
|b(x)| \leq \text { Const }_{a, k} M, \quad x \in \bigcup_{u \in V} X_{u}^{n} \text {. }
$$

Let $u$ be any vertex. Among the triangles attached to $u$, let $\tau=[u, v, w]$ be the one that contains $Y_{u}^{n}$, and let $\tilde{\tau}=[u, v, \tilde{w}]$ be the other triangle attached to the edge $[u, v]$. Since $b \in \Lambda^{\perp}$, we have

$$
b\left(x_{\alpha, \tilde{\tau}}\right)=\sum_{|\beta|=\alpha_{\tilde{w}}}\left(\begin{array}{c}
\alpha_{\tilde{w}} \\
\beta
\end{array}\right) \frac{S_{u}^{\beta_{u}} S_{v}^{\beta_{v}} S_{w}^{\beta_{w}}}{S^{\alpha_{\tilde{w}}}} b\left(x_{\left(\alpha_{u}, \alpha_{v}, 0\right)+\beta, \tau}\right),
$$

where $\alpha=\left(\alpha_{u}, \alpha_{v}, \alpha_{\tilde{w}}\right) \in \mathbb{Z}_{+}^{3}$ with $|\alpha|=k$ and $\alpha_{u} \geq k-\mu$. From (16) we see that

Moreover,

$$
\left|b\left(x_{\left(\alpha_{u}, \alpha_{v}, 0\right)+\beta, \tau}\right)\right| \leq M, \quad \alpha_{u} \geq k-\mu .
$$

This shows that

$$
\left|S_{u}^{\beta_{u}} S_{v}^{\beta_{v}} S_{w}^{\beta_{w}} / S^{\alpha_{\tilde{w}}}\right| \leq \text { Const }_{a} .
$$

$$
\left|b\left(x_{\alpha, \tilde{\tau}}\right)\right| \leq \text { Const }_{a, k} M, \quad \alpha_{u} \geq k-\mu .
$$

Repeating this process, we obtain

$$
|b(x)| \leq \text { Const }_{a, k} M, \quad x \in X_{u}^{n}, n=0,1, \ldots, \mu .
$$

Next, we shall prove (25) for $n=\mu+1, \ldots, 2 r$. If $u$ is a singular interior vertex, this can be done by the same argument as before. On the other hand, if $u$ is a nonsingular vertex, we then prove (25) by induction on $n$ as follows. Let $n$ be an integer in $\{\mu+1, \ldots, 2 r\}$ and assume that $(25)$ holds for $0,1, \ldots, n-$ 1. We wish to prove that (25) also holds for $n$. For this purpose, we shall 
employ the smoothness conditions given in Lemma 2. For a triangle $\tau$ and $\alpha \in \mathbb{Z}_{+}^{3}$ with $|\alpha|=k$, we let $C_{\alpha, \tau}$ be defined as in (5). Let $e=[u, v]$ be an oriented edge attached to $u$, and let $\tau=[u, v, w]$ and $\tilde{\tau}=[u, v, \tilde{w}]$ be the two triangles with common edge $e$. It is assumed that the orientation of $\tau$ agrees with that of $e$. By Lemma 2, we have

$$
C_{(k-n, n-m, m), \tilde{\tau}}=\sum_{\ell=0}^{m} C_{(k-n, n-\ell, \ell), \tau}\left(\begin{array}{c}
m \\
\ell
\end{array}\right) \frac{S_{v}^{m-\ell} S_{w}^{\ell}}{S^{m}},
$$

for $0 \leq m \leq n \leq k$. In order to estimate $C_{\alpha, \tau}$, we introduce $A_{\alpha, \tau}$, as follows. For each triangle $\tau=[u, v, w]$ attached to $u$ and $\alpha=\left(\alpha_{u}, \alpha_{v}, \alpha_{w}\right) \in \mathbb{Z}_{+}^{3}$ with $|\alpha|=k$, let

$$
A_{\alpha, \tau}:=C_{\alpha, \tau} \text { for } 0 \leq \alpha_{v}, \alpha_{w} \leq r .
$$

Moreover, if $\alpha=(k-n, n-\ell, \ell)$ for some $(n, \ell)$ with $\mu+1 \leq n \leq 2 r$ and $2 n-3 r-1 \leq \ell \leq n-r-1$, we will consider

$$
A_{(k-n, n-\ell, \ell), \tau}:=C_{(k-n, n-\ell, \ell), \tau}+\sum_{j=0}^{2 n-3 r-2} a_{\ell j} C_{(k-n, n-j, j), \tau},
$$

where the coefficients $a_{\ell j}$ are to be determined. Fix an integer $j \in\{0,1, \ldots$, $2 n-3 r-2\}$. If $S_{v}=0$, we set $a_{\ell j}:=0$ for all $\ell=2 n-3 r-1, \ldots, n-r-1$; otherwise, let $a_{\ell j}$ be the solutions of the system

$$
\sum_{\ell=2 n-3 r-1}^{n-r-1} a_{\ell j}\left(\begin{array}{c}
m \\
\ell
\end{array}\right)\left(\frac{S_{v}}{S_{w}}\right)^{j-\ell}=\left(\begin{array}{c}
m \\
j
\end{array}\right), \quad m=n-r, \ldots, r,
$$

of linear equations. Since the matrix $\left(\left(\begin{array}{c}m \\ \ell\end{array}\right)\right)_{n-r \leq m \leq r, 2 n-3 r-1 \leq \ell \leq n-r-1}$ is invertible, there exists a unique solution for $\left(a_{\ell j}\right)$. The choice of $\left(a_{\ell j}\right)$ was made in such a way that the equalities

$$
A_{(k-n, n-m, m), \tilde{\tau}}=\sum_{\ell=2 n-3 r-1}^{m} A_{(k-n, n-\ell, \ell), \tau}\left(\begin{array}{c}
m \\
\ell
\end{array}\right) \frac{S_{v}^{m-\ell} S_{w}^{\ell}}{S^{m}}
$$

are valid for all $(m, n)$ with $\mu+1 \leq n \leq 2 r$ and $n-r \leq m \leq r$. Also, we have

$$
\left|a_{\ell j}\left(S_{v} / S_{w}\right)^{j-\ell}\right| \leq \text { Const }_{k} \text {. }
$$

Since $\ell \geq 2 n-3 r-1>j$ and $\left|S_{v} / S_{w}\right| \leq$ Const $_{a, k}$, we obtain

$$
\left|a_{\ell j}\right| \leq \text { Const }_{k}\left|S_{v} / S_{w}\right|^{\ell-j} \leq \text { Const }_{a, k} \text {. }
$$

Next, we define, for convenience,

$$
C\left(x_{\alpha, \tau}\right):=C_{\alpha, \tau}
$$

and

$$
A\left(x_{\alpha, \tau}\right):=A_{\alpha, \tau} \text { for } x_{\alpha, \tau} \in X_{u}^{n}, \mu+1 \leq n \leq 2 r .
$$

Then it follows from (26) that

$$
\sum_{x \in X_{u}^{n}} \lambda(x) A(x)=0 \quad \forall \lambda \in \Lambda_{u}^{n}
$$


Recall from Section 3 that $Z_{u}^{n}$ is a subset of $X_{u}^{n}$ with $\# Z_{u}^{n}=\# \Lambda_{u}^{n}$, such that the inequality (19) holds for any subset $Z$ of $X_{u}^{n}$ with $\# Z=\# \Lambda_{u}^{n}$. Rewrite (27) as

$$
\sum_{x \in Z_{u}^{n}} \lambda(x) A(x)=-\sum_{x \in X_{u}^{n} \backslash Z_{u}^{n}} \lambda(x) A(x),
$$

and apply Cramer's rule to the above system of linear equations to yield

$$
|A(x)| \leq \#\left(X_{u}^{n} \backslash Z_{u}^{n}\right) \max _{y \in X_{u}^{n} \backslash Z_{u}^{n}}|A(y)|, \quad x \in Z_{u}^{n}, \quad \mu+1 \leq n \leq 2 r .
$$

Since $a$ is the smallest angle in $\Delta$, the number of triangles attached to the same vertex is bounded from above by a constant depending only on $a$; hence we have

$$
\#\left(X_{u}^{n} \backslash Z_{u}^{n}\right) \leq \text { Const }_{a, k} .
$$

If $y=x_{\beta, \tau}$ for some $\beta$ with $\beta_{u} \geq k-n$ and $y \notin X_{u}^{n}$, then from the proof of Theorem 1, we see that $y \in \bigcup_{m=0}^{n-1}\left(X_{u, e}^{m} \cup X_{v, e}^{m}\right) \cup Y_{e}$; thus, by the induction hypothesis, we have

$$
|b(y)| \leq \text { Const }_{a, k} M \text {. }
$$

This together with the construction of $C(y)$ and $A(y)$ implies that

$$
|A(y)| \leq \text { Const }_{a, k} M, \quad y \in Y_{u}^{n}=X_{u}^{n} \backslash Z_{u}^{n} .
$$

Therefore, by (28), we obtain

$$
|A(x)| \leq \text { Const }_{a, k} M, \quad x \in Z_{u}^{n} .
$$

Again, by the construction of $C(x)$ and $A(x)$, and by the induction hypothesis, we have

$$
|b(x)| \leq \text { Const }_{a, k} M, \quad x \in Z_{u}^{n} .
$$

This establishes $(25)$ for any nonsingular vertex $u$.

Finally, for $x \in X_{e}^{-}$, it is easily seen from the smoothness conditions across the edge $e$ that

$$
|b(x)| \leq \text { Const }_{a, k} M .
$$

This completes the proof of the theorem.

Now let us establish an equivalence relation between the norm of a spline function and that of its B-net representation.

Lemma 4. Let $f \in S_{k}^{0}(\Delta)$ and $b_{f}$ its B-net representation. Then

$$
\|f\|_{\infty} \leq\left\|b_{f}\right\|_{\infty} \leq \text { Const }_{k}\|f\|_{\infty} \text {. }
$$

Proof. According to the definition of $b_{f}$, we have

$$
f(x)=\sum_{|\alpha|=k} b_{\alpha} B_{\alpha, \tau}(x), \quad x \in \tau,
$$

where $b_{\alpha}:=b_{f}\left(x_{\alpha, \tau}\right)$. Since $B_{\alpha, \tau}$ are nonnegative and $\sum_{|\alpha|=k} B_{\alpha, \tau}(x)=1$ for all $x \in \tau$, it follows that $\|f\|_{\infty} \leq\left\|b_{f}\right\|_{\infty}$.

In order to prove the second inequality in (29), we consider the standard 2simplex $\sigma:=\left\{\left(x_{1}, x_{2}\right): x_{1}, x_{2} \geq 0 ; x_{1}+x_{2} \leq 1\right\}$ and a one-to-one affine mapping $Q$ from $\sigma$ onto $\tau$. Since barycentric coordinates are invariant under 
affine transforms, we have $B_{\alpha, \sigma}(y)=B_{\alpha, \tau}(Q y)$ for all $y \in \sigma$. Thus, it follows from (30) that

$$
f(Q y)=\sum_{|\alpha|=k} b_{\alpha} B_{\alpha, \sigma}(y) .
$$

Since $B_{\alpha, \sigma},|\alpha|=k$, constitute a basis of $\pi_{k}$, we have

$$
\left|b_{\alpha}\right| \leq \text { Const }_{k} \sup _{y \in \sigma}\{|f(Q y)|\} \leq \text { Const }_{k}\|f\|_{\infty} .
$$

This completes the proof of the lemma.

We are now in a position to describe a procedure for constructing a stable basis of $S_{k}^{r, \mu}(\Delta)$. For a given point $x$ in $Y$, it follows from Theorem 1 that there is a unique $B_{x} \in S_{k}^{r, \mu}(\Delta)$ whose B-net representation $b$ satisfies

$$
b(y)= \begin{cases}1, & y=x, \\ 0, & y \in Y \backslash\{x\} .\end{cases}
$$

Theorem 1 also tells us that $\left\{B_{x}: x \in Y\right\}$ constitutes a basis of $S_{k}^{r, \mu}(\Delta)$.

Theorem 4. The basis $\left\{B_{x}: x \in Y\right\}$ of $S_{k}^{r, \mu}(\Delta)$ is stable in the sense that there are two positive constants $K_{1}$ and $K_{2}$ depending only on $k$ and a such that

$$
K_{1} \sup _{x \in Y}\left|c_{x}\right| \leq\left\|\sum_{x \in Y} c_{x} B_{x}\right\|_{\infty} \leq K_{2} \sup _{x \in Y}\left|c_{x}\right| .
$$

This basis is also local in the sense that for any $x \in Y$ there exists a vertex $u$ such that

$$
\operatorname{supp} B_{x} \subseteq \overline{S t}^{\lfloor r / 2\rfloor+1}(u) .
$$

Proof. We first prove (32). Let $f=\sum_{x \in Y} c_{x} B_{x}$. Then the B-net representation $b_{f}$ of $f$ satisfies $b_{f}(x)=c_{x}$ for all $x \in Y$. By Lemma 4 and Theorem 3, we have

$$
\|f\|_{\infty} \leq\left\|b_{f}\right\|_{\infty} \leq \text { Const }_{a, k} \sup _{x \in Y}\left|c_{x}\right|
$$

On the other hand, Lemma 4 implies that

$$
\sup _{x \in Y}\left|c_{x}\right| \leq\left\|b_{f}\right\|_{\infty} \leq \text { Const }_{k}\|f\|_{\infty}
$$

The desired inequality (32) now follows at once from the above estimates.

To prove (33), let $x \in Y$ be arbitrarily chosen. If $x \in Y_{\tau}$ for some triangle $\tau$, then supp $B_{x} \subseteq \tau$. Generally, for a given $x \in Y$, there exists a vertex $u$ such that the barycentric coordinate $\left(\alpha_{u}, \alpha_{v}, \alpha_{w}\right)$ of $x$, with respect to any triangle $[u, v, w]$ with $u$ as a vertex, satisfies $\alpha_{u} \geq \frac{k}{2}$. For two vertices $u$ and $v$ in $\Delta$, we denote by $d(u, v)$ the smallest number of edges among all paths joining $u$ and $v$. We claim that for any positive integer $m \leq 2 r+1-\mu$, if $d(u, v) \geq m$, then the B-net representation $b$ of $B_{x}$ vanishes on $\bigcup_{n=0}^{\mu+m-1} X_{v}^{n}$. This will be proved by induction on $m$. If $m=1$, then for any vertex $v \neq u$, $b$ vanishes on $\bigcup_{n=0}^{\mu} Y_{v}^{n}$; hence, by the smoothness conditions around $v$, we see that $b$ vanishes on $\bigcup_{n=0}^{\mu} X_{v}^{n}$. Let $1<m \leq 2 r+1-\mu$ and assume that our claim has been justified for any positive integer $\ell<m$. We must verify it for $m$. Suppose that $d(u, v) \geq m$ and $d(v, w)=1$. Then $d(u, w) \geq m-1$. 
By the induction hypothesis, we see that $b$ vanishes on $\bigcup_{n=0}^{\mu+m-2}\left(X_{v}^{n} \cup X_{w}^{n}\right)$. If $y \in X \backslash Z_{v}^{\mu+m-1}$ and $\lambda(y) \neq 0$ for some $\lambda \in \Lambda_{v}^{\mu+m-1}$, then we see from the proof of Theorem 1 that $b(y)=0$. Hence, $b$ also vanishes on $Z_{v}^{\mu+m-1}$. This shows that $b$ vanishes on $X_{v}^{\mu+m-1}$, and therefore completes the induction procedure. If $d(v, u) \geq 2 r+2-\mu$ and $d(v, w)=1$, then $b$ vanishes on $\bigcup_{n=0}^{2 r} X_{v}^{n}$ and $\bigcup_{n=0}^{2 r} X_{w}^{n}$. Moreover, if one of $u$ and $v$ is an interior vertex, then $b$ vanishes on $X_{e}^{-}$, where $e$ is the oriented edge joining $v$ and $w$. This shows that $b$ vanishes on the star $\overline{S t}(v)$ of the vertex $v$. Therefore, since $2 r+1-\mu=2 r+1-\left\lfloor\frac{3 r+1}{2}\right\rfloor=\left\lfloor\frac{r+2}{2}\right\rfloor$, we have supp $B_{x} \subseteq \overline{S t}^{\lfloor r / 2\rfloor+1}(u)$.

It only remains to prove part (ii) of Lemma 3. For $f \in C(\Delta)$, let $s \in$ $S_{k}^{0}(\Delta)$ be the spline functions given in the approximation scheme as described in Section 3, and let $b$ be the B-net representation of $s$. By the construction of $T f$, we have

$$
T f(x)=\sum_{y \in Y} b(y) B_{y}(x), \quad x \in \Delta .
$$

Let $\tau$ be a triangle of $\Delta$ with vertex $u$ and $x \in \tau$. Then $B_{y}(x) \neq 0$ only if $d(y, u) \leq\lfloor r / 2\rfloor+2$, or equivalently, $y \in \overline{S t}^{\lfloor r / 2\rfloor+2}(u)=N(u)$. Hence, the number of nonzero terms in (34) is bounded above by Const ${ }_{a, k}$. Moreover, $\left\|B_{y}\right\|_{\infty} \leq$ Const $_{a, k}$ by Theorem 4. Thus, it follows from (34) that

$$
|T f(x)| \leq \text { Const }_{a, k} \max _{y \in N(u) \cap Y}\{|b(y)|\} .
$$

By Lemma 4, we may now conclude that

$$
\max _{y \in N(u) \cap Y}\{|b(y)|\} \leq \text { Const }_{k}\left\|\left.s\right|_{N(u)}\right\|_{\infty} \leq \text { Const }_{k}\left\|\left.f\right|_{N(u)}\right\|_{\infty} .
$$

Combining the above estimates, we obtain the desired result (22).

\section{FINAL REMARKS}

1. Recently, de Boor and Jia [3] proved that the order of approximation of $S_{k}^{r}(\tilde{\Delta})$ for $k \leq 3 r+1$ and the three direction mesh $\tilde{\Delta}$ is at most $k$. Hence, $k=3 r+2$ is the smallest degree for which $S_{k}^{r}(\Delta)$ achieves the optimal approximation order of $k+1$.

2. The main difference between our approach and the previous attempts in [5] and [6] is that the set $Z_{u}^{n}$ for $\Lambda_{u}^{n}$ with the property that assertion (28) holds for all $x \in Z_{u}^{n}, n=\mu+1, \ldots, 2 r$, is obtained by applying (19). Consequently, the dependence of the approximation error on the near-singularity of the triangulation $\Delta$ is eliminated. The price to pay is that the supports of the basis functions, as given in Theorem 4, are necessarily larger than those of the vertex splines in [5].

\section{REFERENCES}

1. C. de Boor, A local basis for certain smooth bivariate pp spaces (C. K. Chui, W. Schempp, and K. Zeller, eds.), Multivariate Approximation IV, Birkhäuser, Basel, 1989, pp. 25-30.

2. C. de Boor and K. Höllig, Approximation power of smooth bivariate pp functions, Math. Z. 197 (1988), 343-363. 
3. C. de Boor and R. Q. Jia, A sharp upper bound on the approximation order of smooth bivariate pp functions, J. Approx. Theory 72 (1993), 24-33.

4. C. K. Chui, Multivariate splines, CBMS Series in Applied Mathematics, no. 54, SIAM, Philadelphia, PA, 1988.

5. C. K. Chui and M. J. Lai, On bivariate super vertex splines, Constr. Approx. 6 (1990), 399-419.

6. D. Hong, On bivariate spline spaces over arbitrary triangulation, Master's Thesis, Zhejiang University, 1987.

7. $\longrightarrow$ Spaces of bivariate spline functions over triangulation, Approx. Theory and Appl. 7 (1991), 56-75.

8. R. Q. Jia, B-net representation of multivariate splines, Ke Xue Tong Bao (A Monthly Journal of Science) 11 (1987), 804-807.

9. J. J. Rotman, An introduction to algebraic topology, Graduate Texts in Math., no. 119, Springer-Verlag, New York, 1988.

10. L. L. Schumaker, Recent progress on multivariate splines, Mathematics of Finite Elements and Applications VI (J. Whiteman, ed.), Academic Press, London, 1991, pp. 535-562.

11. A. Ženišek, Interpolation polynomials on the triangle, Numer. Math. 15 (1970), 283-296.

(C. K. Chui and D. Hong) Center for Approximation Theory, Texas A\&M University, College Station, Texas 77843 78712

Current address (D. Hong): Department of Mathematics, The University of Texas, Austin, Texas

E-mail address: dhong@math.utexas.edu

http://www.ma. utexas.edu

(R. Q. Jia) Department of Mathematics, University of Alberta, Edmonton, Alberta, CANADA T6G 2G1

E-mail address: jia@xihu.math.ualberta.ca 